\title{
Syncope due to non-sustained episodes of Torsade de Pointes associated to androgen-deprivation therapy use: a case presentation
}

\author{
Ximena Morales ${ }^{1^{*}+} \mathbb{0}$, Diego Garnica ${ }^{2 \dagger}$, Daniel ssaza $^{3}$, Nicolas Isaza ${ }^{4}$ and Felipe Durán-Torres ${ }^{5}$
}

\begin{abstract}
Background: Abiraterone is a medication frequently used for metastatic castrate-resistant prostate cancer. We report a case of non-sustained episodes of TdP associated with severe hypokalemia due to androgen-deprivation therapy. Few case presentations describe this association; the novelty lies in the potentially lethal cardiovascular events among cancer patients receiving hormonal therapy.

Case presentation: A 70-year-old male presented with recurrent syncope without prodrome. ECG revealed frequent ventricular ectopy, non-sustained episodes of TdP, and severe hypomagnesemia and hypokalemia. During potassium and magnesium infusion for repletion, the patient underwent temporary transvenous atrial pacing. As part of the work-up, coronary angiography revealed a mild coronary artery disease, and transthoracic echocardiogram showed a moderately depressed ejection fraction. After electrolyte disturbances were corrected, the QT interval normalized, and transvenous pacing was no longer necessary. Abiraterone was discontinued during the admission, and the patient returned to baseline.

Conclusions: Cancer treatment is complex and requires a multidisciplinary approach. We presented a case of nonsustained TdP associated with androgen-deprivation therapy in an elderly patient with mild coronary artery disease and moderately reduced ejection fraction. Close follow-up and increased awareness are required in patients with hormonal treatment, especially in the setting of other cardiovascular risk factors.
\end{abstract}

Keywords: Prostatic neoplasms, Abiraterone acetate, Leuprolide, Androgen-deprivation, Ventricular tachycardia, Hypokalemia, Case presentation

\section{Background}

The use of androgen deprivation therapy represents a milestone in treating both castrate sensitive and castrate-resistant metastatic prostate cancer [1-3]. Dual

*Correspondence: ximena.morales@urosario.edu.co

${ }^{\dagger}$ Ximena Morales and Diego Garnica have contributed equally to this work.

${ }^{1}$ School of Medicine and Health Sciences, Internal Medicine Program, Fundación Cardioinfantil, Universidad del Rosario, Carrera 24 \#63C-69, Bogotá, Colombia

Full list of author information is available at the end of the article pharmacologic therapy with medications such as leuprolide and abiraterone acetate is directed towards minimizing testosterone levels and blocking the androgen receptors [4]. Abiraterone acetate plus prednisone has demonstrated a reduction in all-cause mortality compared to placebo in randomized controlled clinical trials [5].

As is the case with other cancer treatment types, such as chemotherapy, immunotherapy, and radiotherapy, androgen deprivation treatment is associated with cardiovascular complications [6, 7]. Abiraterone is usually 
associated with adverse metabolic effects as hyperglycemia, hyperlipidemia, and hypertension. The latter is related to a strong mineralocorticoid effect, which also produces hypokalemia [8]. To prevent this undesired effect prednisone is prescribed along with abiraterone acetate to reduce the incidence of hyperaldosteronism. Despite the use of prednisone, recent studies have reported an increased incidence of cardiovascular events in real-world patients receiving abiraterone acetate and leuprolide [9]. Furthermore, adverse events as ventricular tachycardia and acquired long QT syndrome-related to abiraterone are scarcely reported. In 2019, the Food and Drug Administration (FDA) published a potential signal of serious risks about abiraterone and is currently evaluating the need for regulatory action; however, no official statement has been released [10].

We report a case of acquired long QT syndrome complicated with non-sustained Torsades de Pointes ventricular tachycardia associated with androgen-deprivation therapy.

\section{Case presentation}

A 70-year-old man with a past medical history of hyperlipidemia, hypertension, and castrate-resistant metastatic prostate cancer, presented to the emergency room with recurrent syncope episodes without prodrome, with some episodes occurring in the decubitus position. Medications included abiraterone acetate $1000 \mathrm{mg}$ BID, monthly $7.5 \mathrm{mg}$ injection of leuprolide, losartan $50 \mathrm{mg}$
BID, and metoprolol succinate $12.5 \mathrm{mg}$ QD. Of note, the patient was not receiving prednisone and did not report any chest pain, palpitations, dyspnea, or any other associated symptoms.

Physical exam was notable for a blood pressure of $148 / 92 \mathrm{mmHg}$, cardiac auscultation with irregular beats. Presentation ECG showed sinus rhythm with a QTc interval of $580 \mathrm{~ms}$ (calculated with Bazett's formula), frequent premature ventricular beats, and short runs of non-sustained TdP. (Figs. 1, 2). Laboratory results showed severe hypokalemia $2.4 \mathrm{mEq} / \mathrm{L}$ (reference range $3.5-5.0 \mathrm{mEq} / \mathrm{L}$ ), severe hypomagnesemia $0.8 \mathrm{mg} / \mathrm{dl}$ (Reference range $1.6-2.6 \mathrm{mg} / \mathrm{dl}$ ), and preserved renal function (creatinine $0.6 \mathrm{mg} / \mathrm{dL}$ and blood urea nitrogen was $10 \mathrm{mg} / \mathrm{dL}$ ) for an estimated GFR of $102 / \mathrm{mL} / \mathrm{min} / 1.73 \mathrm{~m}^{2}$ (calculated by CKD-EPI formula).

The patient was admitted to the coronary care unit for continuous telemetry, electrolyte repletion, and temporary transvenous atrial pacing to suppress ectopy and prevent polymorphic ventricular tachycardia. Transthoracic echocardiogram showed a moderate depressed left ventricular ejection fraction of $38 \%$ with global hypokinesis (Fig. 3). Coronary angiography revealed mild lesions in the left anterior descending and the right coronary artery. The ventricular dysfunction was thought to be secondary to arrhythmia induced cardiomyopathy rather than the primary cause of the arrhythmia. After a thorough review of causes of hypokalemia (including medication review, metabolic alkalosis, and gastrointestinal

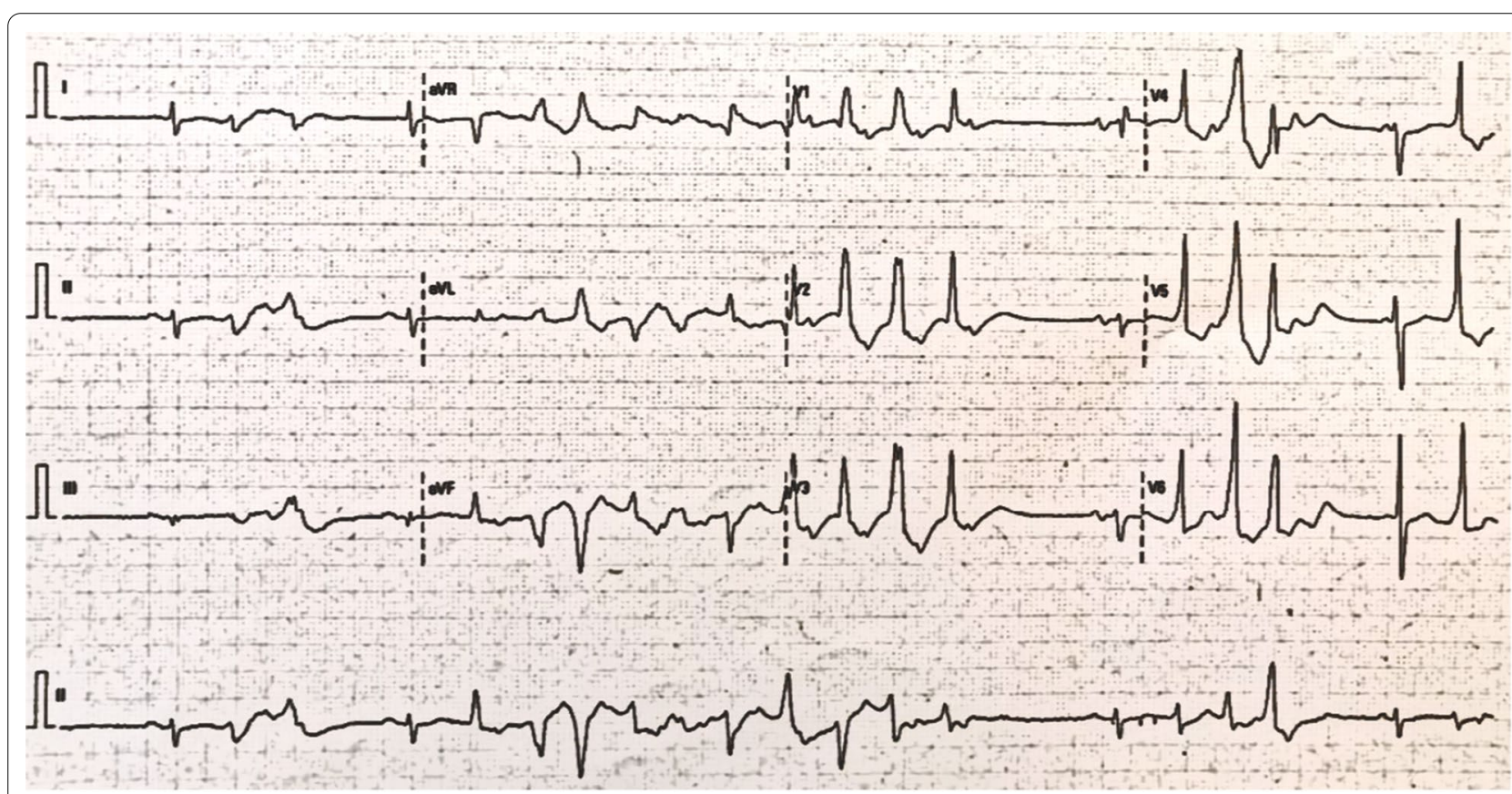

Fig. 1 Non sustained TdP 


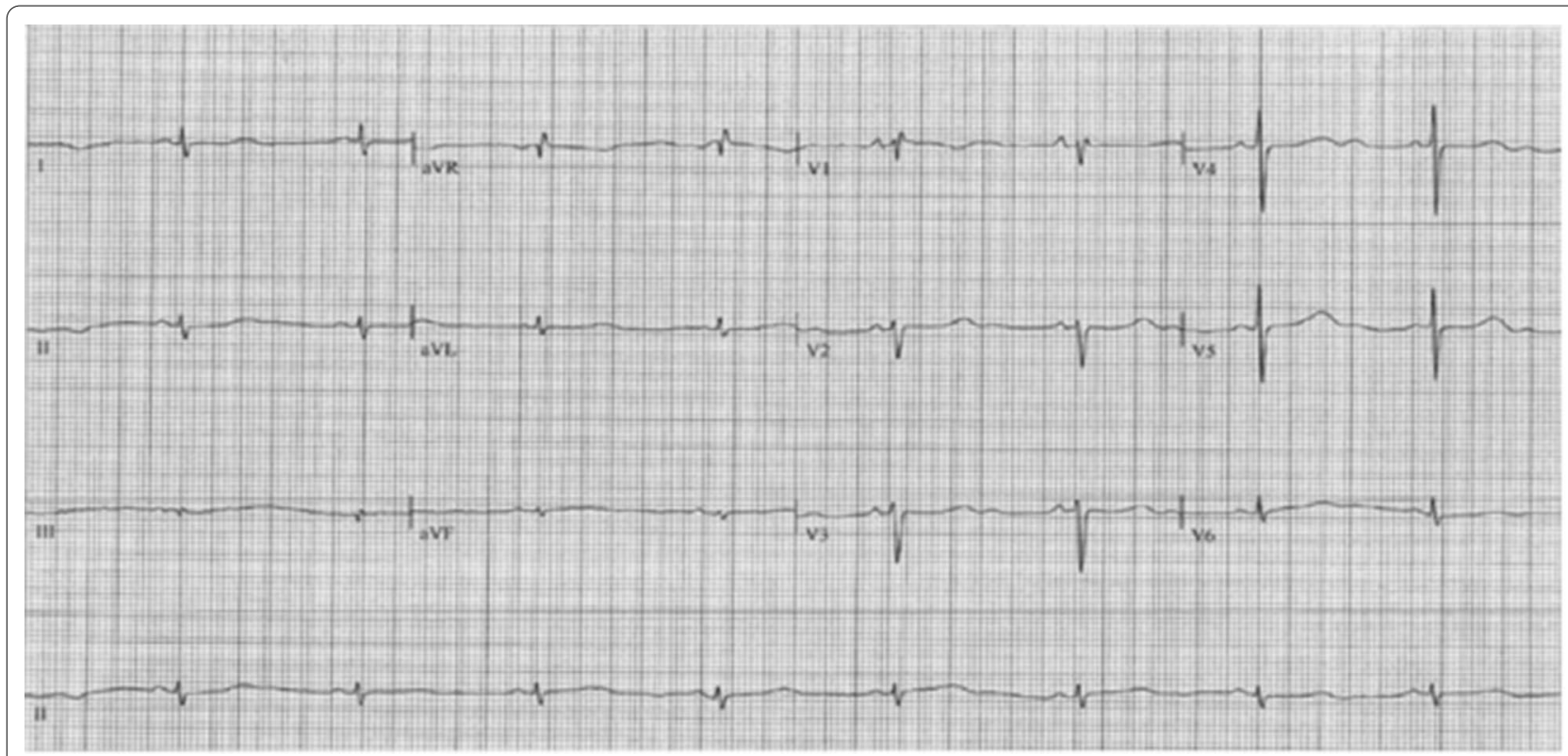

Fig. 2 Sinus rhythm, with long QTc: 526 msg (calculated with Bazett's formula)

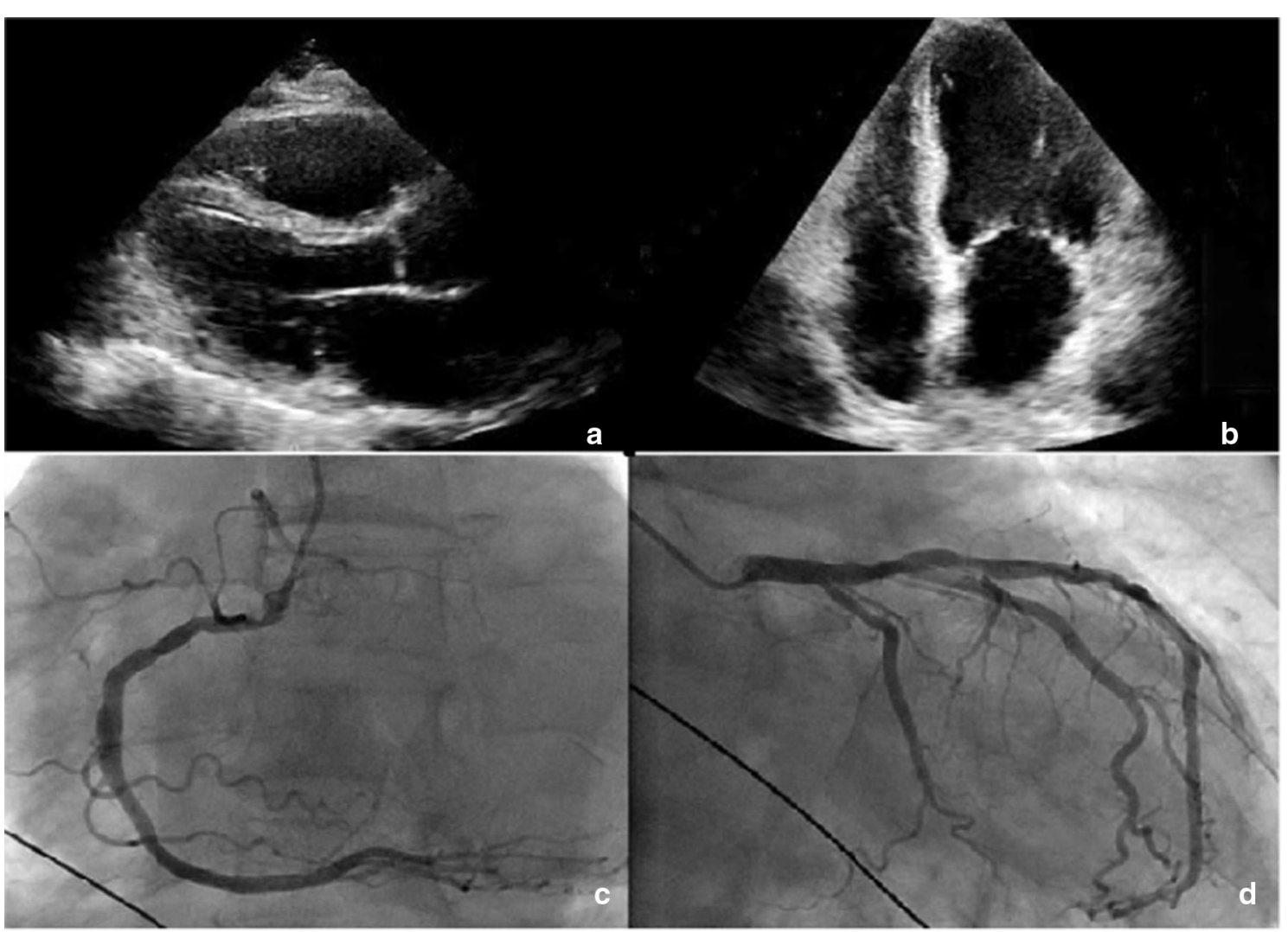

Fig. 3 a, b Transthoracic echocardiogram parasternal long axis view and apical 4 chamber view. $\mathbf{c}$, $\mathbf{d}$ coronary artery angiography showed non-obstructive mild lesions in the left anterior descending artery and the right coronary artery 
losses), abiraterone acetate was suspected to be the cause and was suspended; this was particularly high on the differential given that the patient was not taking prednisone and the use of abiraterone acetate alone can result in a clinical picture similar to hyperaldosteronism. As potassium and magnesium levels normalized, the QTc interval shortened, the ventricular ectopy and runs of non-sustained episodes of TdP were entirely resolved. The patient was eventually transferred to the general ward and was later discharged after three days of being asymptomatic with no telemetry abnormalities.

The patient stopped taking abiraterone acetate and no further electrolyte disturbances were presented, suggesting no underlying genetic abnormalities were the main cause of the arrythmia, such as is the case of Gitelman syndrome, which could have explained the severe hypokalemia and hypomagnesemia (least likely because of the absence of metabolic alkalosis and hypertension medical history).

\section{Discussion and conclusions}

Abiraterone inhibits androgen synthesis and is approved by the FDA for castrate-resistant prostate cancer $[1,2]$. Cancer therapies are known to be associated with cardiovascular and other side effects [6]. In particular, abiraterone causes hypertension and hypokalemia in $32 \%$ and $20 \%$ of cases, respectively $[5,8]$. However, cardiovascular severe adverse events like long QT and ventricular tachycardia are rarely reported $[11,12]$. This cardiovascular event is probably due to the association between androgen deprivation and lower testosterone levels that result in iatrogenic Hypogonadism [13, 14]. A recent study found that testosterone plays an essential role in cardiac repolarization by altering repolarization currents (increasing the repolarizing currents $\mathrm{IKr}$ and IKs, and decreasing the depolarizing current ICaL) [15]. This iatrogenic Hypogonadism induced by medications such as abiraterone and leuprolide has been associated with long QTc and TdP [15], even to the point of suggesting androgen deprivation therapy as the second cause of druginduced long QTc, particularly with abiraterone [16]. It is essential to highlight that leuprolide itself produces and reinforces this hypogonadism state, making an additive effect of these cardiovascular events when it is combined with abiraterone [15].

Androgen deprivation has been associated with cardiotoxicity, including QT prolongation [9, 17, 18]. In 2019, the FDA published a potential signal of serious risks about abiraterone and is currently evaluating the need for regulatory action; nonetheless, no official statement has been released yet [10]. In this setting, we present a case of acquired long QT syndrome complicated with non-sustained Torsades de Pointes ventricular tachycardia associated with severe hypokalemia and hypomagnesemia attributed to the use of abiraterone acetate without prednisone and was enhanced by the concomitant leuprolide use [15]. Furthermore, this acute episode resulted in arrhythmia induced cardiomyopathy that reverted after correction of electrolyte abnormalities and discontinuation of the offending agent. In this particular case, the patient presented with other conditions that are associated with abnormal repolarization and $\mathrm{TdP}$, such as coronary heart disease, hypertension, leuprolide use, male gender and advanced age, suggesting that multiple QTc prolonging phenomena are needed to "hit", before TdP is ensued $[19,20]$. We believe that our case represents a heightened risk of acquired long QTc derived from abiraterone acetate and leuprolide use [15].

Despite having the highest risks due to these medications, the data about the use of abiraterone acetate in men older than 65-years-old is insufficient. Data about adverse events in these specific groups is even more limited because pivotal trials excluded patients with preexisting cardiovascular disorders [5]. Limited evidence includes a cohort study performed between 1991 and 2013 to evaluate if patients with pre-existing cardiovascular disease, using abiraterone or enzalutamide had increased risk of hospitalization and all-cause mortality. In this study, there was an association between hypertension and the use of abiraterone acetate and higher hospitalization rates [18].

More recently, in 2012, a single-arm open clinical trial of 33 patients taking abiraterone evaluated ECG changes during the first two days of use, finding no significant association with QT prolongation [21]; nevertheless, this is a limited study due to trial design, small sample size, and poor ECG follow-up, limiting the ability to conclude. In 2016 a retrospective analysis of abiraterone use in patients with pre-existing cardiovascular conditions concluded that there was no worsening of cardiovascular diseases, and cardiovascular events more frequently reported were fluid overload and hypertension, without reports of arrhythmias during the follow-up [22]. However, this study also presents limitations mainly related to its retrospective nature. In contrast to the previous two studies, a meta-analysis suggests an increased risk in the pooled occurrence of ischaemic heart disease, myocardial infarction, supraventricular tachyarrhythmias, ventricular tachyarrhythmias, heart failure associated with abiraterone acetate [23]. Finally, there are anecdotal reports of abiraterone acetate resulting in QT prolongation and life-threatening ventricular arrhythmias, similar to our case $[11,12]$. Nonetheless, all the studies mentioned above have significant limitations, and further research is required. 
Increased follow-up and awareness are required in patients with androgen deprivation therapy to evaluate the presence of QT prolongation and ventricular arrhythmias arising from electrolyte disorders.

\section{Abbreviations}

TdP: Torsade de Pointes; ECG: Electrocardiogram; QTc: QT interval corrected for heart rate; FDA: Food and Drug Administration; BID: bis in die (which means, in Latin, twice a day); QD: quaque die (which means, in Latin, every day/daily.

\section{Acknowledgments}

Not applicable.

\section{Authors' contributions}

XM collected the data, reviewed the literature, drafted the manuscript contributed to data interpretation, DG reviewed the literature, contributed to data interpretation, and the conception and design of the manuscript, DI extensively revised the manuscript and took care of the patient, NI contributed to data interpretation, FDT extensively revised the manuscript and contributed to data interpretation. All authors read and approved the final manuscript.

\section{Funding}

Not applicable.

\section{Availability of data and materials}

Data sharing does not apply to this article as no datasets were generated or analyzed during the current study.

\section{Declarations}

\section{Competing interests}

All authors declare that they have no competing interests.

\section{Consent for publication}

The patient gave written consent for clinical and personal details along with any identifying images to be published in this manuscript.

\section{Ethics approval and consent to participate}

Not applicable.

\section{Author details}

${ }^{1}$ School of Medicine and Health Sciences, Internal Medicine Program, Fundación Cardioinfantil, Universidad del Rosario, Carrera 24 \#63C-69, Bogotá, Colombia. ${ }^{2}$ Fundación Cardioinfantil, Universidad del Bosque, Bogotá, Colombia. ${ }^{3}$ Division of Cardiology, Fundación Cardioinfantil, Bogotá, Colombia. ${ }^{4}$ Department of Internal Medicine, Beth Israel Deaconess Medical Center, Boston, MA, USA. ${ }^{5}$ School of Medicine and Health Sciences, Public Health Research Group, Universidad del Rosario, Bogotá, Colombia.

Received: 8 September 2020 Accepted: 4 March 2021

Published online: 12 March 2021

\section{References}

1. Cassinello J, Arranz JÁ, Piulats JM, Sánchez A, Pérez-Valderrama B, Mellado $B$, et al. SEOM clinical guidelines for the treatment of metastatic prostate cancer (2017). Clin Transl Oncol Off Publ Fed Span Oncol Soc Natl Cancer Inst Mex. 2018;20(1):57-68.

2. Hoy SM. Abiraterone acetate: a review of its use in patients with metastatic castration-resistant prostate cancer. Drugs. 2013;73(18):2077-91.

3. Schrijvers D. Abiraterone acetate in the treatment of metastatic castration-resistant prostate cancer: review of clinical data. Clin Investig 2012;2(7):707-13.

4. Rehman Y, Rehman Y. Abiraterone acetate: oral androgen biosynthesis inhibitor for treatment of castration-resistant prostate cancer. Drug Des Devel Ther. 2012;6:13-8.
5. Fizazi K, Tran N, Fein L, Matsubara N, Rodriguez-Antolin A, Alekseev BY, et al. Abiraterone plus prednisone in metastatic, castration-sensitive prostate cancer. N Engl J Med. 2017;377(4):352-60.

6. Buza V, Rajagopalan B, Curtis AB. Cancer treatment-induced arrhythmias: focus on chemotherapy and targeted therapies. Circ Arrhythm Electrophysiol. 2017. https://doi.org/10.1161/CIRCEP.117.005443.

7. Porta-Sánchez A, Gilbert C, Spears D, Amir E, Chan J, Nanthakumar K, et al. Incidence, diagnosis, and management of QT prolongation induced by cancer therapies: a systematic review. J Am Heart Assoc. 2017. https://doi. org/10.1161/JAHA.117.007724.

8. Zhu X, Wu S. Risk of hypertension in cancer patients treated with abiraterone: a meta-analysis. Clin Hypertens. 2019;25(1):12.

9. lacovelli R, Ciccarese C, Bria E, Romano M, Fantinel E, Bimbatti D, et al. The cardiovascular toxicity of abiraterone and enzalutamide in prostate cancer. Clin Genitourin Cancer. 2018;16(3):e645-53.

10. Center for Drug Evaluation and Research. October - December 2018 Potential Signals of Serious Risks/New Safety Information Identified by the FDA Adverse Event Reporting System (FAERS). FDA [Internet]. 2019 Dec 20 [cited 2020 Jun 13]; Available from: https://www.fda.gov/drugs/questionsand-answers-fdas-adverse-event-reporting-system-faers/october-decem ber-2018-potential-signals-serious-risksnew-safety-information-identified -fda-adverse

11. Khan A, Kneale B. Life threatening torsades de pointes due to abirateroneinduced hypokaelemia in a patient with metastatic prostate cancer. N Z Med J. 2016;129(1445):124-7.

12. Rodieux F, Nieto N, Sunthorn H, John G, Escher M. Abiraterone acetateinduced life-threatening Torsade de Pointes. Ann Pharmacother. 2015;49(1):152-3

13. Salem J-E, Alexandre J, Bachelot A, Funck-Brentano C. Influence of steroid hormones on ventricular repolarization. Pharmacol Ther. 2016;167:38-47.

14. Hu J-R, Duncan MS, Morgans AK, Brown JD, Meijers WC, Freiberg MS, et al. Cardiovascular effects of androgen deprivation therapy in prostate cancer: contemporary meta-analyses. Arterioscler Thromb Vasc Biol. 2020;40(3):e55-64

15. Lazzerini PE, Bertolozzi l, Acampa M, Cantara S, Castagna MG, Pieragnoli L, et al. Androgen Deprivation Therapy for Prostatic Cancer in Patients With Torsades de Pointes. Front Pharmacol [Internet]. 2020 May 13 [cited 2020 Dec 25];11. Available from: https://www.ncbi.n/m.nih.gov/pmc/articles/ PMC7239032/

16. Salem J-E, Waintraub X, Courtillot C, Shaffer CM, Gandjbakhch E, Maupain $C$, et al. Hypogonadism as a reversible cause of Torsade de Pointes in men. Circulation. 2018:138(1):110-3.

17. Gagliano-Jucá T, Travison TG, Kantoff PW, Nguyen PL, Taplin M-E, Kibel AS, et al. Androgen deprivation therapy is associated with prolongation of QTC interval in men with prostate cancer. J Endocr Soc. 2018;2(5):485-96.

18. Lu-Yao G, Nikita N, Keith SW, Nightingale G, Gandhi K, Hegarty SE, et al. Mortality and hospitalization risk following oral androgen signaling inhibitors among men with advanced prostate cancer by pre-existing cardiovascular comorbidities. Eur Urol. 2020;77(2):158-66.

19. Lazzerini PE, Capecchi PL, El-Sherif N, Laghi-Pasini F, Boutjdir M. Emerging Arrhythmic Risk of Autoimmune and Inflammatory Cardiac Channelopathies. J Am Heart Assoc Cardiovasc Cerebrovasc Dis [Internet]. 2018 Nov 13 [cited 2020 Dec 25];7(22). Available from: https://www.ncbi.n/m.nih.gov/ pmc/articles/PMC6404431/

20. Lazzerini PE, Laghi-Pasini F, Bertolozzi I, Morozzi G, Lorenzini S, Simpatico A, et al. Systemic inflammation as a novel QT-prolonging risk factor in patients with torsades de pointes. Heart. 2017;103(22):1821-9.

21. Tolcher AW, Chi KN, Shore ND, Pili R, Molina A, Acharya M, et al. Effect of abiraterone acetate plus prednisone on the QT interval in patients with metastatic castration-resistant prostate cancer. Cancer Chemother Pharmacol. 2012;70(2):305-13.

22. Verzoni E, Grassi P, Ratta R, Niger M, De Braud F, Valdagni R, et al. Safety of long-term exposure to abiraterone acetate in patients with castrationresistant prostate cancer and concomitant cardiovascular risk factors. Ther Adv Med Oncol. 2016;8(5):323-30.

23. Moreira RB, Debiasi M, Francini E, Nuzzo PV , Velasco GD, Maluf FC, et al. Differential side effects profile in patients with $\mathrm{MCRPC}$ treated with abiraterone or enzalutamide: a meta-analysis of randomized controlled trials. Oncotarget. 2017;8(48):84572-8. 
Publisher's Note

published maps and institutional affiliations.

Springer Nature remains neutral with regard to jurisdictional claims in 